Pacific Journal of Mathematic 


\title{
ON GENERALIZED ELEMENTS WITH RESPECT TO LINEAR OPERATORS
}

\author{
Magnus Giertz
}

\begin{abstract}
Let $X$ be the domain of a linear transformation $A$. Certain subspaces of the second algebraic conjugate $X^{f f}$, obtained by the application of a weak completion process to some suitable subspace of $X$, may be regarded as spaces of generalized elements to which $A$ has a natural extension. When $A$ is a closed Hilbert space transformation, its domain can in this way be extended to a weakly complete space (Theorem 1). For a selfadjoint operator $T$ this extension $\tilde{X}$ may be regarded as the dual of a perfect countably Hilbert space precisely if $T$ has a compact inverse (Theorem 2). Any element in $\tilde{X}$ is obtained by a repeated application of the extended transformation $\widetilde{T}$ to some element in $X$ (Theorem 3). A discussion of the extension of functions of $T$ to $\tilde{X}$, and a spectral theory for $\widetilde{T}$ conclude the paper.
\end{abstract}

The most widely accepted and used method of defining distributions (generalized functions) is probably to regard them as continuous linear functionals over a countably normed space of test functions, i.e. a metric space where the distance function

$$
d(x, y)=\sum_{p=0}^{\infty} 2^{-p} \frac{\|x-y\|_{p}}{1+\|x-y\|_{p}}
$$

is constructed with the aid of an increasing sequence $\left(\|\cdot\|_{p}\right)$ of norms that are pairwise compatible [3]. Other methods are based on completion procedures, analogous to the one used by Cantor to extend the rational number system to the reals $[9,10,11]$, the standard motivation being that in using simpler concepts this makes the theory available to physists, engineers et al. An intuitively appealing way of doing it $[9,20]$ is to start with a linear space $X$ of functions equipped with an inner product, and then consider sequences $\left(x_{n}\right)$ in $X$ with the property that for each $x$ in $X$ the sequence of inner products $\left(x_{n}, x\right)$ converges. The linear space of equivalence classes associated with the obvious equivalence relation for such sequences can be interpreted as an extension $\tilde{X}$ of $X$ obtained by adjoining to this space the missing limit elements of sequences of the type considered. This procedure justifies the use of the term generalized function for an element in $\tilde{X}$.

In $\S 2$ we discuss briefly the obvious extension of this method to the case when we consider sequences in a linear space $X$ for which $\left\langle x_{n}, m\right\rangle$ converges for all $m$ in a total subset $M$ (the set of test functionals) of its algebraic conjugate $X^{f}$. It is natural to regard the 
extension $\tilde{X}(M)$ of $X$ that we obtain as a space of generalized elements to which we may approximate by elements in $X$.

Now the generalized elements represent elements in $M^{f}$, i.e. essentially in $X^{f f}$, rather than in $X^{f}$. Since there exists a canonical embedding of $X$ in $X^{f f}$-but, in general, no such embedding of $X$ in $X^{f}$ - this appears reasonable, and it indicates that the point of view taken in $[9,20]$ is a natural one.

The above method for selecting subspaces of $X^{f f}$ provides a direct and elementary representation of the generalized elements, and in applications to spaces of functions we need only use very simple concepts of integration, etc. But our main reason for adopting it is of a different nature.

Basically, generalized functions are introduced in order to extend the domain of certain differential operators to complete spaces of functions. Now, if we apply the weak completion process considered to the domain $X$ and range $Y$ of a linear operator $A$ we may choose the test functionals so that the sequence $\left(A x_{n}\right)$ defines an element in $\tilde{Y}$ whenever $\left(x_{n}\right)$ defines one in $\tilde{X}$, which gives us an immediate extension of $A$ to all of $\tilde{X}$. So in this paper we regard spaces of generalized elements as extensions of the domains and ranges of linear transformations, determined by the operator or the set of operators we are interested in.

Section 3 deals with transformations with domain and range in a Hilbert space $H$. Given a finite set $\mathscr{A}$ of linear operators in $H$ we shall use the term good element with respect to $\mathscr{A}$ for an element that is in the domain of any polynomial in these transformations and their adjoints, and we denote the subspace of $H$ consisting of good elements by $G_{\mathscr{A}}$ or simply $G$. When $G$ is dense in $H$, it represents a total subspace of the dual $H^{\prime}$, and $\widetilde{G}=\widetilde{G}(G)$ provides an extension of $H$ to which any polynomial in the given operators has a natural extension. The space $\widetilde{G}$ of generalized elements is complete (in the obvious weak sense) if all the transformations are closed (Theorem 1 ). In this situation we may, in fact, equip $G$ with a sequence of inner products so that it becomes countably Hilbert, with a dual that is congruent to $\widetilde{G}$.

The rest of the paper deals with the special case when $\mathscr{A}$ consists of a single self-adjoint operator $T$. The countably Hilbert space $G_{T}$ is then perfect if and only if $T$ has a compact inverse (Theorem 2). After this paper had been submitted for publication a proof of this theorem appeared in a paper by A. Pietsch [14]. Cf. also Notices of the American Mathematical Society, abstract 64T-363 (August 1964) p. 583 .

Furthermore, the transformations 


$$
f_{n}(T)=\left(1+T^{2}+T^{4}+\cdots+T^{2 n}\right)^{1 / 2}, \quad n=1,2, \cdots,
$$

may be extended to $\widetilde{G}_{T}$, and any $\widetilde{x}$ in $\widetilde{G}_{T}$ has the form $\widetilde{x}=f_{n}(\widetilde{T}) x$ for some integer $n$ and some $x$ in $H$. It follows that each generalized element can also be written as $\widetilde{x}=\widetilde{T}^{n} x+y$, where $x$ is in $H$ and $y$ is in $G_{T}$. We may take $y=0$ if zero is not in the spectrum of $T$, and choose $y$ so that $T y=0$ if zero is an isolated point of the spectrum (Theorem 3). When $T$ is a differential operator this is sometimes referred to as the fundamental theorem of distribution theory. For instance, if $T=i(d / d t)$ restricted to absolutely continuous functions of period $2 a$, then $G_{T}$ is the space of infinitely differentiable functions of the same period (usually denoted by $\hat{K}(a)$ ), and every generalized function can in this case be interpreted as the result of repeated differentiation of a continuous periodic function, plus a constant.

The functions $u(T)$ which have an immediate extension to $\widetilde{G}_{T}$ are those which map $G_{T}$ into $G_{T}$. Theorem 4 gives necessary and sufficient conditions on $u$ for this to be the case, and also classifies those functions $u$ for which the extended transformation $u(\widetilde{T})$ maps all of $\widetilde{G}_{T}$ into $G_{T}$.

The extension $\widetilde{E}_{\lambda}$ of the resolution of the identity associated with $T$ is also a resolution of the identity, and it is of the same type as $E_{\lambda}$ in the sense that they are simultaneously discontinuous, of continuous growth, or constant. It is related to $\widetilde{T}$ in much the same way as $E_{\lambda}$ is related to $T$. The nature of the inverse of $\widetilde{T}-\lambda \widetilde{I}$ is, for instance, determined by the behaviour of $\widetilde{E}_{\lambda}$ : the inverse exists precisely if $\lambda$ is a point of continuity, and it is defined on all of $\widetilde{G}_{T}$ precisely if $\lambda$ is a point of constancy. Moreover, $\widetilde{T}$ has the same characteristic vectors as $T$.

The fact that $\widetilde{E}_{\lambda}-\widetilde{E}_{\mu}(-\infty<\mu<\lambda<\infty)$ maps $\widetilde{G}_{T}$ into $G_{T}$ enables us to construct operator Stieltjes integrals of the form $\int_{\alpha}^{\beta} u(\lambda) d \widetilde{E}_{\lambda} \widetilde{x}$ to obtain the representation

$$
u(\widetilde{T}) \widetilde{x}=\left[\left(\int_{-n}^{n} u(\lambda) d \widetilde{E}_{\lambda} \widetilde{x}\right)\right] .
$$

We may interpret this as a method for evaluating certain divergent integrals. If $T$ has a discrete spectrum we have a summation method for divergent series.

When $H$ is represented as a direct integral of Hilbert spaces of functions so that $T$ corresponds to the operator $\Lambda$ defined by multiplication by the independent variable $\lambda$, then $\widetilde{G}_{T}$ is represented by $\widetilde{G}_{A}$. In the special case where $T$ has a simple spectrum we may in most cases choose a generating element $\widetilde{g}$ in $\widetilde{G}_{T}$ for which

$$
\sigma(\lambda)=\left(\left(\widetilde{E}_{2}-\widetilde{E}_{0}\right) \widetilde{g}, \widetilde{g}\right)
$$


has the jump one at each discontinuity, and increases like $\lambda$ at all points of continuous increase. The representation $L_{\sigma}^{2}$ of $H$ is then particularly simple, and the mapping identifying $L_{\sigma}^{2}$ and $H$ is the Fourier-Plancherel transformation when $T=i(d / d t)$ with domain and range in $L^{2}(-\infty, \infty)$.

The last section contains a few simple examples.

2. Extensions of linear spaces. The terminology in this section follows [19]. Let $X$ be a real or complex vector space and $M$ a subset of its algebraic conjugate $X^{f}$. We use $\langle x, m\rangle$ to denote the value of a linear functional $m$ in $X^{f}$ at a point $x$ in $X$, and $\left(x_{n}\right)$ to denote a countably infinite sequence $x_{1}, x_{2}, \cdots$ in $X$. We shall say that $\left(x_{n}\right)$ converges $M$-weakly to $x$ if

$$
\lim _{n \rightarrow \infty}\left\langle x_{n}, m\right\rangle=\langle x, m\rangle
$$

holds for all $m$ in $M$, and that $\left(x_{n}\right)$ is an $M$-weak Cauchy sequence if the limit on the left hand side exists for all $m$ in $M$. We may clearly without loss of generality assume that $M$ is a subspace.

When $M$ is total we obtain an extension $\tilde{X}$ of $X$ by adjoining the missing limit elements of $M$-weak Cauchy sequences, on analogy with the standard procedure for the completion of, say, metric spaces.

The formal definition of $\tilde{X}$ is as follows: Let $R$ be the vector space of $M$-weak Cauchy sequences with addition and scalar multiplication defined in the natural way, and let $N$ be the subspace of $R$ consisting of sequences that converge $M$-weakly to zero. Then

$$
\widetilde{X}=\widetilde{X}(M)=R / N
$$

is the required extension of $X$. The totality of $M$ ensures that the mapping $J: X \rightarrow \widetilde{X}$, defined by

$$
J x=[(x)]
$$

is one-to-one, so $J$ embeds $X$ isomorphically in $\tilde{X}$. We shall use $\widetilde{x}$ as a general symbol for an element $\left[\left(x_{n}\right)\right]$ in $\tilde{X}$, but we shall also often keep the notation $x$ for an element of the form $J x$.

The concepts of $M$-weak Cauchy sequence and $M$-weak convergence carry over to $\widetilde{X}$ if the functionals in $M$ are extended in the obvious way:

$$
\langle\widetilde{x}, m\rangle=\lim _{n \rightarrow \infty}\left\langle x_{n}, m\right\rangle .
$$

We shall say that $\tilde{X}$ is ( $M$-weakly) complete if every $M$-weak Cauchy sequence is $M$-weakly convergent. In general $\tilde{X}$ is not complete.

To obtain a counterexample, let $X$ be the real vector space of 
continuous real-valued functions defined on the real line, with pointwise addition and scalar multiplication. Then the set $M$ of all linear functionals $\delta_{a}$, "evaluation at the point $a$ ", of the form

$$
\left\langle x, \delta_{a}\right\rangle=x(a),
$$

where $a$ is a real number, and $x(\alpha)$ denotes the value of the function $x$ at the point $a$, is total. The $M$-weak Cauchy sequences are in this case sequences of continuous functions converging pointwise, and $\widetilde{X}(M)$ can in a natural way be identified with the Baire class 1. $M$-weak convergence in $\tilde{X}(M)$ is then equivalent to pointwise convergence, and it is well known that the Baire class 1 is not complete with respect to this convergence.

The reason for the above construction of $\tilde{X}$ is that it provides us with a possibility of extending linear operators in a direct and simple manner. Let $A$ be a linear transformation from $X_{1}$ into $X_{2}$ and let $A^{T}$ denote the transpose of $A$. Then if $M_{1}$ and $M_{2}$ are total subspaces of $X_{1}^{f}$ and $X_{2}^{f}$, respectively, and if $M_{1}$ is chosen so that it contains $A^{T} M_{2}$, we may define $\widetilde{A}: \widetilde{X}_{1}\left(M_{1}\right) \rightarrow \widetilde{X}_{2}\left(M_{2}\right)$ by

$$
\widetilde{A} \widetilde{x}=\left[\left(A x_{n}\right)\right] .
$$

The equality $\left\langle A x_{n}, m_{2}\right\rangle=\left\langle x_{n}, A^{T} m_{2}\right\rangle$ implies that the definition is consistent.

Our main objective is to extend linear operators to complete spaces. We have therefore tried (without success) to find a general classification of the sets $M$ for which $\tilde{X}(M)$ is complete. It is easy to prove that this is the case if $M$ is spanned by at most denumerably many functionals, but this is of no help as far as the applications we have in mind are concerned. We can, however, achieve the desired result for operators that are defined and closed in a Hilbert space, and fortunately this is very often the case.

Before we turn to inner product spaces, let us just briefly mention a topological formulation of the problem of completeness.

For an arbitrary subset $S$ of $M^{f}$, we define ps $S$, the pseudo $M$-closure of $S$, to be the set of all $z$ in $M^{f}$ for which there exists a sequence $\left(z_{n}\right)$ in $S$ such that $\lim _{n \rightarrow \infty}\left\langle m, z_{n}\right\rangle=\langle m, z\rangle$ holds for all $m$ in $M$, and we say that $S$ is $M$-closed if $\operatorname{ps} S=S$. Note that $\operatorname{ps} A$ is not necessarily $M$-closed, and that ps $S$ and $S(M)$ coincide only if $M$ is total regarded as a subset of $S^{f}$.

The set of $M$-closed subsets of $M^{\rho}$ are the closed sets of a topology of $M^{f}$. This $M$-topology is in general not linear, but it is translation invariant, and scalar multiplication is continuous in the two variables separately.

Since $\tilde{X}(M)=\operatorname{ps} X$ the question of $M$-weak completeness now 
becomes the question whether or not ps $X$ is equal to the $M$-closure of $X$. This would, for instance, be the case if the $M$-topology is a metric topology, or if, in the terminology of [17], the topology for $M^{f}$ defined by the mapping $S \rightarrow$ ps $S$ satisfies axiom $F$.

From now on we assume that an inner product is defined on $X$. We may then regard $X$ as a dense subspace of a Hilbert space $H$. We shall use the standard notation $(x, y)$ for the value of the inner product of $x$ and $y$ in $H$. Any subset $Y$ of $H$ corresponds in a natural way to a subset of $X^{f}$, and as such it is total precisely if $Y$ is dense in $H$. For simplicity, we shall make no notational distinction between $Y$ and its natural embedding in $X^{s}$.

We shall consider extensions of $H$ of the form $\tilde{X}(Y)$, where $Y$ is dense in $H$. In this situation we use the notation $(\widetilde{x}, y)$ for $\langle\widetilde{x}, y\rangle$ $\left(=\lim _{n \rightarrow \infty}\left(x_{n}, y\right)\right)$ and define $\left.(y, \widetilde{x})=\overline{(\widetilde{x}, y}\right)$. Whenever $\left(h_{n}\right)$ is a $Y$-weak Cauchy sequence in $H$ we can choose a sequence $\left(x_{n}\right)$ in $X$ with the property that

$$
\lim _{n \rightarrow \infty}\left(x_{n}, y\right)=\lim _{n \rightarrow \infty}\left(h_{n}, y\right)
$$

for all $y$ in $Y$. This means that the extensions (with respect to the same $Y$ ) of all dense subspaces of a Hilbert space are equivalent. More precisely, if $c$ denotes the natural embedding of $\tilde{X}$ in $Y^{s}$ we have $\iota \widetilde{X}(Y)=\iota \tilde{H}(Y)$. The only difference between them is that an equivalence class in $\widetilde{H}(Y)$ contains more sequences than the corresponding one in $\widetilde{X}(Y)$. It is by changing $Y$ that we obtain essentially different extensions.

To illustrate, we take for $H$ the space $L^{2}(-\infty, \infty)$, and for $Y$ the Schwartz class $S$ [cf. 9]. If $f$ is a locally square integrable function with the property that $\left(1+t^{2}\right)^{-n} f$ is in $L^{2}(-\infty, \infty)$ for some integer $n$, then there certainly exists an $\tilde{f}$ in $\widetilde{L}^{2}(S)$ such that

$$
(\tilde{f}, g)=\int_{-\infty}^{\infty} f(t) \overline{g(t)} d t
$$

holds for all $g$ in $S$. Hence there is such an element in $\widetilde{S}(S)$ as well [9, pp. 22-23].

If $Z$ is a subset of $Y$, then any $Y$-weak Cauchy sequence in $X$ is also a $Z$-weak Cauchy sequence. But this does not imply that $\tilde{X}(Z)$ is larger than $\tilde{X}(Y)$. No inclusion relation $\iota \tilde{X}(Y) \subseteq \iota \widetilde{X}(Z)$ exists, for different elements in $\iota \tilde{X}(Y)$ may correspond to the same element in $\iota \widetilde{X}(Z)$. Note that $\iota \widetilde{X}(H)=\iota H$ since $H$ is weakly complete, i.e. the choice $Y=H$ gives us no proper extension.

Let $A$ be a linear transformation from $X$ into $H$. If the domain $D_{A^{*}}$ of its adjoint $A^{*}$ is dense in $H$ we may extend $A$ to an operator from $\tilde{X}(Y)$ into $\tilde{H}(Z)$ by taking for $Z$ a dense subset of $D_{A^{*}}$ and for 
$Y$ any dense subset of $H$ containing $A^{*} Z$. If there exists a dense subspace $G$ of $X$ with the property that both $A$ and $A^{*}$ map $G$ into $G$, we can choose $Y=Z=G$ to obtain an extension $\tilde{A}: \widetilde{X}(G) \rightarrow \tilde{H}(G)$. Since $\iota \widetilde{G}=\iota \widetilde{X}=\iota \widetilde{H}$, and $\widetilde{A} x=A x$ for all $x$ in $X$, it is only a formal change to regard $\widetilde{A}$ as an operator from $\widetilde{G}(G)$ into $\widetilde{G}(G)$. But this change enables us to give a direct definition of the extension $\widetilde{A}^{n}$ of $A^{n}$ for $n=1,2, \cdots$. We can, in fact, extend any transformation $B$, such that both $B$ and $B^{*}$ map $G$ into $G$, to a transformation $\widetilde{B}$ from $\widetilde{G}$ into $\widetilde{G}$ by putting

$$
\widetilde{B} \widetilde{x}=\left[\left(B x_{n}\right)\right]
$$

for each $\widetilde{x}=\left[\left(x_{n}\right)\right]$ in $\widetilde{G}$. It is clear that $\widetilde{B}$ satisfies $(\widetilde{B})^{n}=\widetilde{B}^{n}$, and $(\widetilde{B} \widetilde{x}, y)=\left(\widetilde{x}, B^{*} y\right)$ holds for all $y$ in $G$. Also, $\widetilde{B}$ is sequentially continuous in the sense that $\widetilde{B} \widetilde{x}_{n}$ converges $G$-weakly to $\widetilde{B} \widetilde{x}$ whenever $\widetilde{x}_{n}$ converges $G$-weakly to $\widetilde{x}$.

3. Extensions of linear operators in a Hilbert space. The terminology in this section follows $[3,5]$. Let $\left\{A_{i}\right\}_{i=1}^{m}$ be a finite set of linear operators with domain and range in a Hilbert space $H$. By a good element with respect to the set $\left\{A_{i}\right\}$ we shall mean an element that is in the domain of any product of these operators and their adjoints. We denote by $G=G_{A_{1}, A_{2}, \ldots, A_{m}}$ the subspace of $H$ consisting of all good elements with respect to $\left\{A_{i}\right\}$. Thus any one of the transformations $A_{i}$ or $A_{i}^{*}, i=1,2, \cdots, m$, maps $G$ into $G$. It might of course happen that $G$ contains only the zero element, but very often $G$ is dense in $H$. When this is the case we can define $\widetilde{G}=\widetilde{G}(G)$, and extend each transformation $A_{i}$-in fact each polynomial in these operators and their adjoints-to this space. We shall refer to the elements in $\widetilde{G}$ as generalized elements with respect to the set $\left\{A_{i}\right\}$. It turns out that $\widetilde{G}$ is complete if each transformation is closed in $H$. In order to show this we equip $G$ with a sequence of inner products so that it becomes countably Hilbert, with an adjoint that is congruent to $\widetilde{G}$.

Theorem 1. Let $A_{i}, i=1,2, \cdots, m$, be a finite set of closed linear operators with domain and range in a Hilbert space $H$. Assume that the subspace $G$ of good elements with respect to these operators is dense in $H$. Then $\widetilde{G}$ is (G-weakly) complete.

Proof. For each nonnegative integer $p$, let $A_{p k}, k=1,2, \cdots,(2 m)^{p}$, denote the operators that appear in the expansion of $\left(\sum_{i=1}^{m} A_{i}+A_{i}^{*}\right)^{p}$. Put

$$
(x, y)_{n}=\sum_{p=0}^{n} \sum_{k=1}^{(2 m ; p}\left(A_{p k} x, A_{p k} y\right), \text { and } G_{n}=\bigcap_{k=1}^{(2 m)^{n}} D_{A_{n k}} \text {, for } n=0,1,2, \cdots
$$


The verification that $(\cdot, \cdots)_{n}$ defines an inner product on $G_{n}$ is straight forward, and it is clear that the norms $\|\cdot\|_{n}=(\cdot, \cdot)_{n}^{1 / 2}$ are nondecreasing in $G$. We shall show that they are also compatible, and that $G_{n}$, with the inner product $(\cdot, \cdot,)_{n}$, is a Hilbert space.

Assume that $\left(x_{\nu}\right)$ is a Cauchy sequence in $G_{n+1}$ which-regarded as a sequence in $G_{n}$-converges to $x$ in $G_{n}$. Then $\left(A_{n k} x_{\nu}\right)_{\nu=1}^{\infty}$ converges to $A_{n k} x$ in $H$ for $k=1,2, \cdots,(2 m)^{n}$. The equality

$$
\left\|x_{\nu}\right\|_{n+1}^{2}=\left\|x_{\nu}\right\|_{n}^{2}+\sum_{k=1}^{(2 m) n+1} \sum_{i=1}^{m}\left(\left\|A_{i} A_{n k} x_{\nu}\right\|^{2}+\left\|A_{i}^{*} A_{n k} x_{\nu}\right\|^{2}\right)
$$

shows that for each $i=1,2, \cdots, m ; k=1,2, \cdots,(2 m)^{n+1}$, the sequences $\left(A_{i} A_{n k} x_{\nu}\right)_{\nu=1}^{\infty}$ and $\left(A_{i}^{*} A_{n k} x_{\nu}\right)_{\nu=1}^{\infty}$ are Cauchy sequences in $H$ and converge accordingly in $H$. Since each operator $A_{i}$ and $A_{i}^{*}, i=1,2, \cdots, m$, is closed, we conclude that $x$ must be in $G_{n+1}$, and that $\left(x_{\nu}\right)$ converges to $x$ in this space. It follows by induction that the norms are compatible, and that $G_{n}$ is complete. Since

$$
G=\bigcap_{n=0}^{\infty} G_{n}
$$

this implies that $G$ is a countably Hilbert space [3, Chapter I §3.2]. Now let $c$ be the natural embedding of $\widetilde{G}$ in $G^{f}$, defined by $\langle y, \iota \widetilde{x}\rangle=(y, \widetilde{x})$ for all $y$ in $G$. Then $c$ certainly maps $G$ into $G^{\prime}$. Since $G^{\prime}$ is weakly complete [3, Chapter I $\left.\S 5.6\right]$, and

$$
\langle y, \iota \widetilde{x}\rangle=\lim _{n \rightarrow \infty}\left\langle y, \iota x_{n}\right\rangle
$$

we conclude that $c$ actually maps $\widetilde{G}$ into $G^{\prime}$. All we have to do now is to verify that $c$ is onto $G^{\prime}$. But we know [3, Chapter I, $\S 4.3$ ] that for every $x^{\prime}$ in $G^{\prime}$ there exists an integer $n$ and an $x$ in $G_{n}$ for which

$$
\left\langle y, x^{\prime}\right\rangle=(y, x)_{n}=\left(y, \sum_{p=0}^{n} \sum_{k=1}^{(2 m)^{p}} \widetilde{A}_{p k}^{*} A_{p k} x\right)=(y, \widetilde{x}),
$$

with $\widetilde{x}$ in $\widetilde{G}$. This completes the proof of Theorem 1 .

Whenever we speak of the space of good elements with respect to a set of closed linear operators we shall mean the countably Hilbert space $G$ constructed in Theorem 1 . Note that if $\left(x_{n}\right)$ converges to zero in $G$, then $\left(A_{i} x_{n}\right), i=1,2, \cdots, m$, tends to zero as well. This means that the restriction of each operator $A_{i}$ to $G$ is continuous in $G$.

The last lines of the proof give us the following

CoRollary. Let $\left\{A_{i}\right\}_{i=1}^{m}$ be a finite set of closed linear operators in a Hilbert space $H$, and let $G$ be the subspace of good elements with respect to there operators. Then for every $\widetilde{x}$ in $\widetilde{G}$ there exist 
an index $n$ and an element $x$ in $G_{n}$ such that

$$
\widetilde{x}=\sum_{p=0}^{n} \sum_{k=1}^{(2 m) p} \widetilde{A}_{p k}^{*} A_{p k} x=\sum_{p=0}^{n} \widetilde{T}_{p} x,
$$

where $\widetilde{T}_{0}=I$ and $\widetilde{T}_{p+1}=\sum_{i=1}^{m}\left(\widetilde{A}_{i} \widetilde{T}_{p} A_{i}^{*}+\widetilde{A}_{i}^{*} \widetilde{T}_{p} A_{i}\right)$.

To illustrate, let $\left\{\varphi_{\nu}, \nu=0,1,2, \cdots\right\}$ be an orthonormal basis in a separable Hilbert space $H$, and let $\left(k_{\nu}\right)$ be a sequence of positive real numbers that tends monotonically to infinity. Define a "torsion operator" $A$ by letting the domain of $A$ consist of all $x=\sum_{\nu=0}^{\infty} c_{\nu} \varphi_{\nu}$ in $H$ for which $\sum_{\nu=0}^{\infty}\left|c_{\nu} k_{\nu}\right|^{2}$ converges, and then take $A x=\sum_{\nu=0}^{\infty} c_{\nu} k_{\nu} \varphi_{\nu+1}$. $A$ is closed, and $G=G_{A}$ consists of all $x=\sum c_{\nu} \varphi_{\nu}$ in $H$ for which the sum

$$
\sum_{\nu=0}^{\infty}\left|k_{\nu} k_{\nu+1} \cdots k_{\nu+p} c_{\nu}\right|^{2}
$$

converges for all $p$. A straightforward calculation shows that for any $y$ in $G$ the operators $T_{p}$ of the corollary above give

$$
\sum_{p=0}^{n} T_{p} y=\sum_{\nu=0}^{\infty} \tau_{\nu}(n) c_{\nu} \varphi_{\nu},
$$

where the $\tau_{\nu}$ 's are real numbers satisfying

$$
1<\frac{\tau_{\nu}(n)}{k_{\nu}^{2} k_{\nu+1}^{2} \cdots k_{\nu+n-1}^{2}}<2^{n+1}, \quad n=1,2, \cdots .
$$

Thus for any $\widetilde{x}$ in $\widetilde{G}$ there exist an index $n$ and an $x=\sum a_{\nu} \varphi_{\nu}$ in $G_{n}$ such that for any $y=\sum b_{\nu} \varphi_{\nu}$ in $G$

$$
(\widetilde{x}, y)=\left(x, \sum_{p=0}^{n} T^{p} y\right)=\sum_{\nu=0}^{\infty} a_{\nu} \tau_{\nu}(n) \bar{b}_{\nu}=\sum_{\nu=0}^{\infty} c_{\nu}(n) \bar{b}_{\nu} .
$$

Putting $y=\varphi_{\nu}$ we see that $c_{\nu}(n)=\left(\widetilde{x}, \varphi_{\nu}\right)$, which means that

$$
\widetilde{x}=\left[\left(\sum_{\nu=0}^{n}\left(\widetilde{x}, \varphi_{\nu}\right) \varphi_{\nu}\right)\right] \text {. }
$$

It follows from the inequalities for the $\tau_{\nu}$ 's that

$$
\left[\left(\sum_{\nu=0}^{n} c_{\nu} \varphi_{\nu}\right)\right] \in \widetilde{G} \Leftrightarrow \sum_{\nu=0}^{\infty}\left|\frac{c_{\nu}}{k_{\nu} k_{\nu+1} \cdots k_{\nu+p}}\right|^{2}
$$

converges for some $p$.

4. Generalized elements with respect to a self-adjoint trans. formation. The terminology in this paragraph follows [1]. Let $T$ be a self-adjoint operator in a Hilbert space $H$, and let $E_{\lambda}$ be the 
corresponding resolution of the identity. We adopt the convention that $E_{\lambda-0}=E_{\lambda}$. Functions of $T$ are defined as in [1, §74]. The good elements with respect to $T$ are those for which the integral

$$
\int_{-\infty}^{\infty}|\lambda|^{n} d\left(E_{\lambda} x, x\right)
$$

is finite for all natural numbers $n . \quad G_{T}$ is dense in $H$ since it contains, for instance, the domain of the densely defined transformation exp $|T|$. The sequence of inner products giving the topology in $G_{T}$ may be defined by

$$
(x, y)_{n}=\sum_{p=0}^{n}\left(T^{p} x, T^{p} y\right)=\left(f_{n}(T) x, f_{n}(T) y\right),
$$

where $f_{n}(\lambda)=\left(1+\lambda^{2}+\lambda^{4}+\cdots+\lambda^{2 n}\right)^{1 / 2}$. We might here equally well use any other sequence $\left(f_{n}\right)$ of real-valued and, say, continuous functions satisfying

$$
1 \leqq f_{1}(\lambda) \leqq f_{2}(\lambda) \leqq \cdots \leqq f_{n}(\lambda) \leqq f_{n+1}(\lambda) \leqq \cdots
$$

and

$$
\lim _{|\lambda| \rightarrow \infty}|\lambda|^{-n} f_{n}(\lambda)=1, \quad n=1,2, \cdots .
$$

Whatever sequence of this type we choose, the integral

$$
\int_{-\infty}^{\infty}|\lambda|^{p} f_{n}^{2}(\lambda) d\left(E_{\lambda} y, y\right)
$$

is finite for any $y$ in $G_{T}$ and all natural numbers $n$ and $p$. This means that $f_{n}(T)$ maps $G_{T}$ into $G_{T}$, and thus the corollary of Theorem 1 takes the form: $\widetilde{x}$ is a generalized element with respect to $T$ if and only if $\widetilde{x}=f_{n}(\widetilde{T}) x$ for some natural number $n$ and some $x$ in $H$.

Countably Hilbert spaces that are perfect or nuclear have several interesting properties [cf. 3 and 5]. There is a very simple classification of the $G_{T}$-spaces that have these properties: $G_{T}$ is perfect if and only if the resolvent operator $R_{\lambda}=(\lambda-T)^{-1}$ is compact for some $\lambda$, and $G_{T}$ is nuclear if and only if $R_{\lambda}^{n}$ is of Hilbert-Schmidt type for some $\lambda$ and some natural number $n$.

THEOREM 2. $G_{T}$ is perfect if and only if $T$ has a pure point spectrum with no finite limit point, and $G_{T}$ is nuclear if and only if it is perfect and in addition

$$
\sum_{\lambda_{\nu} \neq 0}\left|\lambda_{\nu}\right|^{-p} m_{\nu}
$$

converges for some $p$, where $m_{\nu}$ is the multiplicity of the characteristic value $\lambda_{\nu}$. 
Proof. We shall first show that $G_{T}$ cannot be perfect if the spectrum of $T$ has a limit point at a finite value of $\lambda$, say $\lambda_{0}$. For if this is the case then the projection $E_{\lambda_{2}}-E_{\lambda_{1}}$, where $\lambda_{1}<\lambda_{0}<\lambda_{2}$, is of infinite rank, which means that we can choose an infinite orthonormal sequence $\left(y_{\nu}\right)=\left(\left(E_{\lambda_{2}}-E_{\lambda_{1}}\right) x_{\nu}\right)$ in $H$. The set $B=\left\{y_{\nu}\right\}$ can obviously contain no accumulation point in $H$-much less in $G_{T}$. But $B$ is bounded in $G_{T}$ since

$$
\left\|y_{\nu}\right\|_{p}^{2}=\left\|f_{p}(T) y_{\nu}\right\|^{2}=\int_{\lambda_{1}}^{\lambda_{2}}\left|f_{p}(\lambda)\right|^{2} d\left\|E_{\lambda} y_{\nu}\right\|^{2} \leqq \max _{\lambda_{1} \leqq \lambda \leqq \lambda_{2}}\left|f_{p}(\lambda)\right|^{2}=K_{p},
$$

so $G_{T}$ is not perfect.

Now assume that $T$ has a pure point spectrum with the only limit point at infinity. Choose a number $\mu$ in the resolvent set of $T$ and put $S=S(T)=c(T-\mu I)$, where the constant $c$ is chosen so that $S(\lambda)$ is greater than one for all $\lambda$ in the spectrum of $T$. We may then consider the inner products in $G_{T}$ defined by $(x, y)_{n}=\left(S^{n} x, S^{n} y\right)$. Thus if $B$ is a bounded infinite subset of $G_{T}$ containing the sequence $\left(x_{\nu}\right)$, we know that for each $n$ the set $\left\{\left\|S^{n} x_{\nu}\right\|\right\}_{\nu=1}^{\infty}$ is bounded. Taking $n=1$, and noting that $S^{-1}$ is compact, we conclude that $\left(x_{\nu}\right)$ must contain a subsequence $\left(x_{0 \nu}\right)$ converging in $H$ to $x_{0}$, say. And if we take $n=2$ it follows that $\left(S x_{0 \nu}\right)$ contains a subsequence $\left(S x_{1 \nu}\right)$ that converges in $H$. Since $x_{1 \nu}$ converges to $x_{0}$ and $S$ is closed it follows $x_{0}$ is in $D_{s}$ and that $\left(S x_{1 \nu}\right)$ converges to $S x_{0}$.

Continuing this process we obtain a sequence of subsequences $\left(x_{n \nu}\right)_{\nu=1}^{\infty}, n=0,1,2, \cdots$, with the property that $\left(S^{n} x_{n \nu}\right)$ converges to $S^{n} x_{0}$ in $H$. But then the diagonal sequence $\left(x_{\nu \nu}\right)$ converges to $x_{0}$ in $G_{T}$, i.e. $x_{0}$ is an accumulation point of $B$. We have now proved the first part of the theorem.

As for the second part, we know that $G_{T}$ is perfect if it is nuclear, so in this case $T$ must have a point spectrum with infinity as the only limit point. Denote the characteristic values by $\lambda_{\nu}, \nu=$ $0,1, \cdots$, let $\left\{\phi_{\nu}\right\}_{\nu=0}^{\infty}$ be a complete orthonormal set of characteristic elements of $T$, and let $\mu_{\nu}$ be the characteristic value corresponding to $\varphi_{\nu}$ (several $\mu_{\nu}$ 's may be equal). Put $\varphi_{\nu}^{(n)}=f_{n}^{-1}\left(\mu_{\nu}\right) \varphi_{\nu}$ for $n=1,2, \cdots$. Then $\left\{\varphi_{\nu}^{(n)}\right\}_{\nu=0}^{\infty}$ is an orthonormal basis for the Hilbert space $G_{n}$ of elements in the domain of $f_{n}(T)$, with the inner product $(\cdot, \cdots)_{n}$. But for any nonnegative integers $n$ and $m$, and any $y$ in $G_{n}$, we have

$$
y=\sum_{\nu=0}^{\infty}\left(y, \varphi_{\nu}\right) \varphi_{\nu}=\sum_{\nu=0}^{\infty} \frac{f_{m}\left(\mu_{\nu}\right)}{f_{n}\left(\mu_{\nu}\right)}\left(y, \varphi_{\nu}^{(n)}\right)_{n} \varphi_{\nu}^{(m)} .
$$

Choosing $m=0$ we see that the assuption that $G_{T}$ is nuclear implies that $\sum_{\nu=0}^{\infty}\left[f_{p}\left(\mu_{\nu}\right)\right]^{-1}$ must converge for some $p$, which in turn implies that 


$$
\sum_{\lambda_{\nu} \neq 0} m_{\nu}\left|\lambda_{\nu}\right|^{-p}
$$

converges. Conversely, if this condition is fulfilled we can for every $m$ find an $n$ so that the sum

$$
\sum_{\nu=0}^{\infty} \frac{f_{m}\left(\mu_{\nu}\right)}{f_{n}\left(\mu_{\nu}\right)}
$$

converges. It follows from $(*)$ that $G_{T}$ is nuclear, and the proof is complete.

Our next theorem states that any generalized element with respect to $T$ is the result of a repeated application of $\widetilde{T}$ to some element in $H$, plus a good element having the character of a constant or nearconstant (with respect to $T$ ).

In some applications where $T$ is a differential operator this gives us a representation of $\widetilde{G}_{T}$ as a class of derivatives, and it is then often referred to as the "fundamental theorem of distribution theory".

Theorem 3. Any $\widetilde{x}$ in $\widetilde{G}_{T}$ can be written as

$$
\tilde{x}=\widetilde{T}^{n} x_{1}+x_{2},
$$

where $x_{1}$ is in $H, x_{2}$ is in $G_{T}$, and $n$ is a natural number. We may omit $x_{2}$ if $\lambda=0$ is not in the spectrum of $T$. If $\lambda=0$ is an isolated point of the spectrum we can as $x_{2}$ take an element with $T x_{2}=0$, and if $\lambda=0$ belongs to the continuous spectrum we may, given any $\varepsilon>0$, choose $x_{2}$ so that $\left\|T x_{2}\right\|<\varepsilon$.

Proof. Let $\widetilde{x}=f_{n}(\widetilde{T}) x$ be an element of $\widetilde{G}_{T}$, and let $\alpha$ be a positive number that is not a characteristic value of $T$. Define $g(\lambda)$ by

$$
g(\lambda)= \begin{cases}f_{n}(\lambda) & \text { if }-\alpha<\lambda \leqq \alpha \\ 0 & \text { otherwise }\end{cases}
$$

and $h(\lambda)$ by $g(\lambda)+\lambda^{n} h(\lambda)=f_{n}(\lambda)$. Then $g(T)$ maps $H$ into $G_{T}$, and $h(T)$ maps $H$ into $H$ since it is a bounded transformation. But then

$$
\widetilde{x}=f_{n}(\widetilde{T}) x=\widetilde{T}^{n} h(T) x+g(T) x=\widetilde{T}^{n} x_{1}+x_{2},
$$

where $x_{1}$ is in $H$ and $x_{2}$ in $G_{T}$. Since

$$
\left\|x_{2}\right\|^{2}=\int_{-\alpha}^{\alpha} f_{n}^{2}(\lambda) d\left(E_{\lambda} x, x\right), \quad \text { and } \quad\left\|T x_{2}\right\|^{2}=\int_{-\alpha}^{\alpha} \lambda^{2} f_{n}^{2}(\lambda) d\left(E_{\lambda} x, x\right)
$$

we see that $x_{2}=0$ if $[-\alpha, \alpha]$ contains no point of the spectrum and $T x_{2}=0$ if the interval contains no other point of the spectrum than $\lambda=0$. Finally, if $\lambda=0$ is in the continuous spectrum we can make 
$\left\|T x_{2}\right\|$ smaller than any given positive $\varepsilon$ by choosing $\alpha$ small enough, since

$$
\left\|T x_{2}\right\|^{2} \leqq \alpha^{2}\|x\|^{2} \max _{|\lambda| \leqq \alpha} f_{n}^{2}(\lambda)
$$

5. Extensions of functions of a self-adjoint transformation. Any transformation $u(T)$ which maps all of $G_{T}$ into $G_{T}$ can be extended to $\widetilde{G}_{T}$ in a direct manner by the definition $u(\widetilde{T}) \widetilde{x}=\left[\left(u(T) x_{n}\right)\right]$. In this case the simple relations $u(\widetilde{T})+v(\widetilde{T})=(u+v)(\widetilde{T})$ and $u(\widetilde{T}) v(\widetilde{T})=$ $(u v)(\widetilde{T})$ follow automatically. A function $c(T)$ that does not map $G_{T}$ into $G_{T}$ can also be extended, but now the domain of the extension becomes a proper subspace of $\widetilde{G}_{T}$. For the domain of $c(\widetilde{T})$ we take all $\widetilde{x}$ in $\widetilde{G}_{T}$ that are of the form $f_{n}(\widetilde{T}) x$ with $x$ in the domain of $c(T)$, and for such $\tilde{x}$ we define

$$
c(\widetilde{T}) \widetilde{x}=f_{p}(\widetilde{T}) c(T) x .
$$

Here the domain of $c(\widetilde{T})$ contains no element in $H$ that is not already in the domain of $c(T)$, so there is nothing particularly good about the elements in $G_{T}$ as far as $c(T)$ is concerned. In order to extend $c(T)$ to $H$ we should instead consider the good elements with respect to this transformation.

The following theorem provides a classification of those transformations that can be extended to all of $\widetilde{G}_{T}$, and also of those whose extension maps all of $\widetilde{G}_{T}$ back into $G_{T}$.

THEOREM 4. Let $u$ be a complex valued function of a real variable $\lambda,-\infty<\lambda<\infty$. Introduce the conditions

(i) $u$ is locally $L_{\sigma}^{2}$ for all $\sigma(\lambda)=\left(E_{\lambda} x, x\right)$ with $x$ in $H$,

(ii) the essential limit of $|\lambda|^{p} u(\lambda)$ as $\lambda$ tends to infinity on the spectrum of $T$ is zero.

Then the following holds true:

(a) The transformation $u(T)$ maps $G_{T}$ into $G_{T}$ if and only if $u$ satisfies (i), and (ii) for some (negative) integer $p$.

(b) The transformation $u(\widetilde{T})$ maps $\widetilde{G}_{T}$ into $G_{T}$ if and only if $u$ satisfies (i), and (ii) for all integers $p$.

Proof. We prove statement (b) first. If $u(\widetilde{T})$ maps $\widetilde{G}_{T}$ into $G_{T}$, then $\left(f_{p} u\right)(T)$ is a bounded transformation from $H$ into $H$ for any $p=0,1,2, \cdots$. This means that $u$ must be in all the $L_{\sigma}^{2}$ 's in question and that $|\lambda|^{p} u(\lambda)$ is bounded for all $p$ and therefore converges to zero for all $p$ when $|\lambda| \rightarrow \infty$. Conversely, assume that these condi. tions hold and let $\widetilde{x}=\left[\left(x_{n}\right)\right]$ be any element in $\widetilde{G}_{T}$. Then $\lambda^{p} u(\lambda)$ is bounded for all $p$ and therefore the transformation $T^{p} \bar{u}(T)$ maps $H$ 
into $G_{T}$ for $p=0,1,2, \cdots$. But then

$$
\lim _{n \rightarrow \infty}\left(T^{p} u(T) x_{n}, z\right)=\lim _{n \rightarrow \infty}\left(x_{n}, T^{p} \bar{u}(T) z\right)
$$

exists for any $z$ in $H$. Since $H$ is weakly complete it follows that $T^{p} u(\widetilde{T}) \widetilde{x}$ is in $H$ for all $p$, i.e. that $u(\widetilde{T}) \widetilde{x}$ is in $G_{T}$.

As for the statement (a) the "if" part is obvious. To prove that the given conditions are also necessary we first note that if there exists a finite interval $[\alpha, \beta]$ and an $x$ in $H$ so that $\int_{\alpha}^{\beta}|u(\lambda)|^{2} d\left(E_{\lambda} x, x\right)$ does not exist, then the good element $\left(E_{\beta}-E_{\alpha}\right) x$ is not in the domain of $u(T)$, so this transformation cannot map $G_{T}$ into $G_{T}$.

Finally, if $\lambda^{-p} u(\lambda)$ does not essentially converge to zero with respect to some $\sigma(\lambda)$ as $\lambda \rightarrow+\infty$ for any integer $p$, we can inductively choose sets $S_{k}$ of positive $\sigma$-measure on the positive real axis, each one to the right of the preceding ones, so that $|u(\lambda)|>\lambda^{k}$ on $S_{k}$. But then

$$
v(\lambda)= \begin{cases}|u(\lambda)|^{-1 / 2} & \text { on } \bigcup_{k=1}^{\infty} S_{k} \\ 0 & \text { otherwise }\end{cases}
$$

has the property that $\lambda^{p} v(\lambda)$ converges to zero as $\lambda$ tends to infinity for all $p$, so $v(T)$ maps $H$ into $G_{T}$. But $u(T) v(T)$ is unbounded, so its domain cannot be all of $H$. This means that $u(T)$ cannot be defined on all of $G_{T}$, and the proof is complete.

It is easy to check that the extension $\widetilde{E}_{\lambda}$ of the resolution of the identity associated with $T$ is also a resolution of the identity. It follows, for instance, from the representation $\widetilde{x}=f_{n}(\widetilde{T}) x$ that $\widetilde{E}_{\lambda}$ converges (weakly) to the identity transformation on $\widetilde{G}_{T}$, since

$$
\lim _{\lambda \rightarrow \infty}\left(\widetilde{E}_{\lambda} \widetilde{x}, y\right)=\lim _{\lambda \rightarrow \infty}\left(E_{\lambda} x, f_{n}(T) y\right)=(\widetilde{x}, y) .
$$

The verification that $\widetilde{E}_{\lambda}$ is of the same type as $E_{\lambda}$, in the sense that they are both constant, of continuous growth, or discontinuous at the same values of $\lambda$, presents no problem.

These observations indicate that $\widetilde{T}$ is a natural extension of $T$. It turns out that most anything that can be said about $T$ in $H$ in fact also holds for $\widetilde{T}$ in $\widetilde{G}_{T}$. The price we pay is, of course, that it holds only $G_{T}$-weakly. We now proceed to give some additional support to this statement, and begin by nothing that $\widetilde{T}$ and $T$ have the same characteristic values and the same characteristic vectors. Clearly $T x=\lambda x$ in $H$ implies that $\widetilde{T} x=\lambda x$ in $\widetilde{G}_{T}$. Conversely, assume that $\widetilde{T} \widetilde{x}=\lambda \widetilde{x}$ holds in $\widetilde{G}_{T}$. Choosing $n$ in the representation $\widetilde{x}=f_{n}(\widetilde{T}) x$ so large that $x$ is in the domain of $T$ we then have $\left((T-\lambda) x, f_{n}(T) y\right)=0$ 
for all $y$ in $G_{T}$. Since $f_{n}(T)$ has a bounded inverse mapping $G_{T}$ into $G_{T}$ this means that $(T x-\lambda x, y)=0$ for all $y$ in $G_{T}$, i.e. that $T x=\lambda x$ in $H$. So $\lambda$ is a characteristic value of $T$, and $x$ is a corresponding characteristic element. But then $x$ is in $G_{T}$ and $\widetilde{x}=f_{n}(T) x=f_{n}(\lambda) x$ is, of course, also a characteristic element.

Now the theorem [5, Ch. I, $\S 4.5$ Th. $\left.5^{\prime}\right]$ stating that any self-adjoint transformation in a rigged Hilbert space has a complete system of characteristic elements (in the weak sense we are discussing) implies that $G_{T}$ cannot be nuclear unless $T$ already has a complete set of characteristic elements in $H$ (cf. Theorem 2). Although the extension of $T$ to $\widetilde{G}_{T}$ introduces no new characteristic elements, we may of course get such elements if we extend transformations mapping $G_{T}$ into $G_{T}$ that are not functions of $T$. [Example $2, \S 6]$.

We also note that if $E(\Delta)=E_{\lambda}-E_{\mu}$ (or $=E_{\lambda+0}-E_{\lambda}$ ), then $\widetilde{E}(\Delta)$ and $E(\Delta)$ have the same range. In fact, for any $y$ in the range of $\widetilde{E}(\Delta)$ we have

$$
y=\widetilde{E}(\Delta) \widetilde{x}=E(\Delta) \widetilde{E}(\Delta) \widetilde{x}=E(\Delta) z,
$$

where $z$ is in $G_{T}$. Moreover, $\mu$ is a point of continuity of $\widetilde{E}_{\lambda}$ if and only if $\widetilde{T}-\mu \widetilde{I}$ is one-to-one and also a point of constancy if and only if the range of $\widetilde{T}-\mu \widetilde{I}$ is all of $\widetilde{G}_{T}$. The direct construction of operator Stieltjes integrals as a limit of Stieltjes sums [cf. 1] carries over from $H$ to $\widetilde{G}_{T}$. In fact, let $\left(S_{n}\right)$ be a sequence of Stieltjes sums converging (strongly) in $H$ to $\int_{\alpha}^{\beta} c(\lambda) d E_{\lambda}$, and let $\widetilde{S}_{n}$ be the sum obtained by changing $E\left(\Delta_{k}\right)$ to $\widetilde{E}\left(\Delta_{k}\right)$. Then $\widetilde{S}_{n}$ maps $\widetilde{G}_{T}$ into $G_{T}$, and $\left(\widetilde{S_{n}} \widetilde{x}\right)$ is a $G_{T}$-weak Cauchy sequence. Letting $\left[\left(\widetilde{S}_{n} \widetilde{x}\right)\right]$ define $\int_{\alpha}^{\beta} c(\lambda) d \widetilde{E_{\lambda}} \widetilde{x}$ we have

$$
\int_{\alpha}^{\beta} c(\lambda) d \widetilde{E}_{\lambda} \widetilde{x}=c(\widetilde{T})\left(\widetilde{E}_{\beta}-\widetilde{E}_{\alpha}\right) \widetilde{x}
$$

and thus also

$$
c(\widetilde{T}) \widetilde{x}=\left[\left(\int_{-n}^{n} c(\lambda) d \widetilde{E}_{2} \widetilde{x}\right)\right] .
$$

Theorems of the type: " $x$ is in the domain of $c(T)$ if and only if $c$ is in $L_{\sigma}^{2}$ with $\sigma(\lambda)=\left(E_{2} x, x\right)$ " may be formulated in $\widetilde{G}_{T}$ with the slight modification that we have to use $\sigma(\lambda)=\left(\left(\widetilde{E}_{2}-\widetilde{E}_{0}\right) \widetilde{x}, \widetilde{x}\right)$ instead. It is then easily verified that $\widetilde{x}$ is in the domain of $c(\widetilde{T})$ if and only if $c f_{n}^{-1}$ is in $L_{\sigma}^{2}$ for some $n$.

Finally, the realization of $H$ as a direct integral of Hilbert spaces in which $T$ corresponds to multiplication by the "independent variable" give us a realization of $\widetilde{G}_{T}$ as a space of generalized elements with respect to multiplication. Let us just mention a few results in the special case when $T$ is cyclic (i.e. has a simple spectrum). Then any 
generating element $h$ in $H$ is also a generating element in $\widetilde{G}_{T}$, and so is $\widetilde{g}=f_{p}(\widetilde{T}) h$ for any $p$, meaning that to any $\widetilde{x}$ in $\widetilde{G}_{T}$ there corresponds a transformation $c(\widetilde{T})$ such that $\widetilde{x}=c(\widetilde{T}) \widetilde{g}$. In fact, $\widetilde{x}=f_{n}(\widetilde{T}) x$ and $x=\varphi(T) h$ imply that $\widetilde{x}=\left(f_{n} \varphi f_{p}^{-1}\right)(\widetilde{T}) f_{p}(\widetilde{T}) h=c(\widetilde{T}) \widetilde{g}$.

The mapping $\widetilde{x} \rightarrow c(\lambda)$ gives us the desired realization of $\widetilde{G}_{T}$ as the space of generalized elements with respect to the transformation $\Lambda$ in $L_{\sigma}^{2}$, where $\sigma(\lambda)=\left(\left(\widetilde{E}_{\lambda}-\widetilde{E}_{0}\right) \widetilde{g}, \widetilde{g}\right)$ and $\Lambda$ is defined by $(\Lambda c)(\lambda)=$ $\lambda c(\lambda)$. It is easy to verify that

$$
\begin{gathered}
c(\widetilde{T}) \widetilde{g} \in \widetilde{G}_{T} \Leftrightarrow c \in \widetilde{G}_{A} \\
c(\widetilde{T}) \widetilde{g} \in H \Leftrightarrow c \in L_{\sigma}^{2} \\
c(\widetilde{T}) \widetilde{g} \in G_{T} \Leftrightarrow c \in G_{\Lambda} .
\end{gathered}
$$

The first statement, for instance, is true because $c(\widetilde{T}) \widetilde{g} \in \widetilde{G}_{T} \Leftrightarrow \widetilde{g}$ is in the domain of $c(\widetilde{T}) \Leftrightarrow c f_{n}^{-1} \in L_{\sigma}^{2}$ for some $n \Leftrightarrow c \in \widetilde{G}_{1}$.

The point of interest here is that we may choose the generating element $\widetilde{g}$ in $\widetilde{G}_{T}$ in such a way that $\sigma$ becomes normalized, in the sence that it has the jump 1 at each point of discontinuity and increases like $\lambda$ at all points of continuous increase. This can, in fact, be done whenever there is a generating element $h$ in $H$ for which

$$
\frac{1}{\lambda} \int_{0}^{\lambda} f_{u}^{2}(\mu) d\left(E_{\mu} h, h\right)
$$

increases to infinity with $\lambda$ for some $n$. Such normalizing generating elements have sometimes been introduced as "improper elements". [Cf. $1, \S 77$ where the normalizing generating elements for the transformations multiplication by $t$ and $i(d / d t)$ in $L^{2}(-\infty, \infty)$ are adjoined to this space].

When $\sigma$ is normalized it is appropriate to refer to the mapping identifying $\widetilde{G}_{T}$ and $\widetilde{G}_{A}$ as the Fourier transform [Example 3, §6]. The equality

$$
(\widetilde{x}, y)=(c(\widetilde{T}) \widetilde{g}, b(\widetilde{T}) \widetilde{g})=\int_{-\infty}^{\infty} c(\lambda) \overline{b(\lambda)} d \sigma(\lambda)
$$

holding for any $\widetilde{x}=c(\widetilde{T}) \widetilde{g}$ in $\widetilde{G}_{T}$, and any $y=b(\widetilde{T}) \widetilde{g}$ in $G_{T}$ generalizes Parseval's relation. In particular the "coefficient function" $c$ in $\widetilde{x}=$ $c(\widetilde{T}) \widetilde{g}$ satisfies the relation

$$
\int_{0}^{\lambda} c(\mu) d \sigma(\mu)=\left(\widetilde{x},\left(\widetilde{E}_{\lambda}-\widetilde{E}_{0}\right) \widetilde{g}\right) .
$$

6. Examples. We conclude this paper with a few applications of the results obtained above.

ExAmple 1. Let $H$ be a separable Hilbert space, and $T$ a self- 
adjoint transformation in $H$ with a simple spectrum having infinity as the only limit point. The points of the spectrum are denoted by $\lambda_{\nu}, \nu=1,2, \cdots$, and the corresponding normalized characteristic elements by $\varphi_{\nu}$. They form a complete orthonormal set in $H$. We may regard this set as given, and then define $T$ in the following manner: Choose any sequence $\left(\lambda_{\nu}\right)$ of real numbers such that $\left|\lambda_{\nu}\right|$ tends to infinity with $\nu$, then take for $D_{T}$ all $x$ in $H$ for which $\sum\left|\lambda_{\nu}\left(x, \varphi_{\nu}\right)\right|^{2}$ converges, and put $T x=\sum \lambda_{\nu}\left(x, \varphi_{\nu}\right) \varphi_{\nu}$ for such $x$. According to Theorem $2, G_{T}$ is perfect. It is also nuclear if $\sum\left|\lambda_{\nu}\right|^{-n}$ converges for some $n$.

The normalizing generating element in $\widetilde{G}_{T}$ is $\widetilde{g}=\sum_{\nu=1}^{\infty} \varphi_{\nu}$, and $\sigma(\lambda)=\left(\left(\widetilde{E}_{\lambda}-\widetilde{E}_{0}\right) \widetilde{g}, \widetilde{g}\right)$ is a montonically increasing step function that is zero at $\lambda=0$ and has the step one at each characteristic value $\lambda_{\nu}$. The representation $c(\widetilde{T}) \widetilde{x}=\int_{-\infty}^{\infty} c(\lambda) d \widetilde{E}_{\lambda} \widetilde{x}$ here takes the form

$$
c(\widetilde{T}) \widetilde{x}=\sum_{\nu=1}^{\infty} c\left(\lambda_{\nu}\right)\left(\widetilde{x}, \varphi_{\nu}\right) \varphi_{\nu},
$$

meaning that

$$
(c(\widetilde{T}) \tilde{x}, y)=\sum_{\nu=1}^{\infty} c\left(\lambda_{\nu}\right)\left(\widetilde{x}, \varphi_{\nu}\right)\left(\varphi_{\nu}, y\right)
$$

holds for all $y$ in $G_{T}$. The representation $\widetilde{x}=f_{n}(\widetilde{T}) x$ tells us that if $\widetilde{x}$ is in $\widetilde{G}_{T}$ then

$$
\sum_{\lambda_{\nu} \neq 0}\left|\lambda_{\nu}^{-n}\left(\widetilde{x}, \varphi_{\nu}\right)\right|^{2}
$$

converges for some $n$, and, conversely, that $\sum c_{\nu} \varphi_{\nu}$ is in $\widetilde{G}_{T}$ if $\sum\left|\lambda_{\nu}^{-n} c_{\nu}\right|^{2}$ converges for some $n$. This can be interpreted as a summation method for certain divergent series in $H$. The coefficient sequence $\left(c_{\nu}\right)$ is, in the terminology of $\S 5$, the inverse Fourier transform of $\sum c_{\nu} \varphi_{\nu}$.

ExAmple 2. Let $q$ be a real valued, positive, infinitely differentiable, and even function of $t,-\infty<t<\infty$, that increases to infinity with $t$. Let $T$ be the self-adjoint transformation $-\left(d^{2} / d t^{2}\right)+q$ with domain and range in $L^{2}(-\infty, \infty)$. Then $T$ is of the type considered in Example 1, and $G_{T}$ is nuclear. Assuming that $q$ satisfies a few additional conditions, not affecting its rate of increase [6, 7], it can be verified that $G_{T}$ is identical with the space of good elements with respect to the two operators differentiation and multiplication by $q$ in $L^{2}$, and that it also coincides with the space $K\left\{M_{p}\right\}$, with $M_{p}=q^{p}$, defined by [cf. 3]

$$
K\left\{q^{p}\right\}=\left\{y ;(q(t))^{p} \frac{d^{n} y}{d t^{n}} \in L^{2}(-\infty, \infty) \text { for } n \leqq p, p=0,1,2, \cdots\right\} .
$$


The choice $q(t)=t^{2}$ is of special importance since the characteristic elements (the Hermite functions $\varphi_{\nu}, \nu=0,1,2, \cdots$ ) are then also characteristic elements of the Fourier transform. In this case the space $G_{T}$ is identical to the Schwartz class $S$, and the representation

$$
\widetilde{x}=\left[\left(\sum_{\nu=0}^{n}\left(\widetilde{x}, \varphi_{\nu}\right) \varphi_{\nu}\right)\right]
$$

gives us an expansion of the generalized functions in $\widetilde{S}$ in series of Hermite functions [8, 21, 22].

Let us now for a moment consider the operators $P, Q$, and $U$, where $P$ and $Q$ are the self-adjoint transformations $i(d / d t)$ and multiplication by $t$ with suitable domains in $L^{2}(-\infty, \infty)$, and $U$ is the Fourier-Plancherel transformation. They all map $G_{T}$ into $G_{T}$. Neither $P$ nor $Q$ has any characteristic elements in $L^{2}(-\infty, \infty)$, but any real number $\lambda$ is a characteristic value of their extensions $\widetilde{P}$ and $\widetilde{Q}$ to $\widetilde{G}_{T}$. The corresponding characteristic vectors are, respectively,

$$
e^{-i \lambda t}=\left[\left(e^{-\left(t^{2} / n\right)-i \lambda t}\right)\right] \text { and } \widetilde{\delta}_{\lambda}=\left[\left(\sqrt{\frac{n}{\pi}} e^{-n(t-\lambda)^{2}}\right)\right] .
$$

The resolutions of the identity associated with $P$ and $Q$ do not map $G_{T}$ into $G_{T}$, so they cannot be extended to all of $\widetilde{G}_{T}$. The extension $\widetilde{U}$ of the Fourier transform to $\widetilde{G}_{T}$ has the same characteristic values as $U$ does, namely the solutions of $\lambda^{4}=1$. But we do obtain additional characteristic vectors. Each element $\widetilde{x}$ of the form

$$
\widetilde{x}=\sum_{\nu=0}^{\infty} c_{4 \nu+k} \varphi_{4 \nu+k}, \quad k=0,1,2,3,
$$

where $\sum_{\nu=0}^{\infty}\left|(2 \nu+1)^{-n} c_{4 \nu+k}\right|^{2}$ converges for some $n$, is a characteristic vector corresponding to the characteristic value $(-i)^{k}$. The representation of an arbitrary element $\widetilde{x}$ in $\widetilde{G}_{T}$ as the sum of four characteristic elements of $\widetilde{U}$ thus carries over from $L^{2}$ to $\widetilde{G}_{T}=\widetilde{S}[15, \S 113]$. The resolution of the identity $E_{2}^{\prime}$ belonging to $U$ maps $G_{T}$ into $G_{T}$, since it is composed of third degree polynomials in $U$. However, the range of $\widetilde{E}^{\prime}(\Delta)$ is, as we have just seen, not equal to that of $E^{\prime}(\Delta)$.

EXAMPLE 3 . Let $P$ be the self-adjoint transformation corresponding to the differential operator $i(d / d t)$ with domain and range in $L^{2}(-\infty, \infty)$ -i.e. restricted to those functions which are absolutely continuous on any finite interval. The spectrum of $P$ is simple, and consists of the entire real axis, so $G_{P}$ is not perfect. Among the elements in $\widetilde{G}_{P}$ we find $\delta$-functions, in fact

$$
\widetilde{\delta}_{a}=\frac{1}{2}\left(\widetilde{I}+\widetilde{T}^{2}\right) e^{-|t-a|}
$$


has the property $\left(x, \widetilde{\delta}_{\alpha}\right)=x(\alpha)$.

For any real numbers $\alpha$ and $\beta$ the projection $\widetilde{E}(\Delta)=\widetilde{E}_{\beta}-\widetilde{E}_{\alpha}$ maps $\widetilde{G}_{P}$ into $G_{P}$, and the value of the good function $\widetilde{E}(\Delta) \widetilde{x}$ at the point $t$ is given by $(\widetilde{E}(\Delta) \widetilde{x})(t)=\left(\widetilde{x}, z_{t}\right)$, where $z_{t}$ is the good function

$$
z_{t}(\alpha, \beta, s)=\frac{e^{i \beta(t-s)}-e^{i \alpha(t-s)}}{2 \pi i(t-s)} .
$$

To see this, we note that certainly $(E(\Delta) y)(t)=\left(y, z_{t}\right)$ holds for any $y$ in $G_{P}[1, \S 77]$, and thus

$$
\left(y, z_{t}\right)=\left(E(\Delta) y, \widetilde{\delta}_{t}\right)=\left(y, \widetilde{E}(\Delta) \widetilde{\delta}_{t}\right)
$$

implies that $z_{t}(\alpha, \beta, s)=\left(\widetilde{E}(\Delta) \widetilde{\delta}_{t}\right)(s)$. But this proves our statement since $(\widetilde{E}(\Delta) \widetilde{x})(t)=\left(\widetilde{E}(\Delta) \widetilde{x}, \widetilde{\delta}_{t}\right)=\left(\widetilde{x}, z_{t}\right)$.

The function $e^{-|t|}$ is a generating element in $L^{2}$, so

$$
\widetilde{g}=\sqrt{2 \pi} \widetilde{\delta}_{0}=\sqrt{\frac{\pi}{2}}\left(\widetilde{I}+\widetilde{T}^{2}\right) e^{-|t|}
$$

is also a generating element. It normalizes $\sigma(\lambda)=\left(\left(E_{\lambda}-E_{0}\right) \widetilde{g}, \widetilde{g}\right)$ since

$$
\left(\left(\breve{E}_{\lambda}-\widetilde{E}_{0}\right) \widetilde{g}\right)(t)=\sqrt{2 \pi} \bar{z}_{t}(0, \lambda, 0)=\frac{e^{-i \lambda t}-1}{-\sqrt{2 \pi} i t}
$$

implies that $\sigma(\lambda)=\lambda$. So in this case $L_{\sigma}^{2}=L^{2}(-\infty, \infty)$.

Thus every $\widetilde{x}$ in $\widetilde{G}_{P}$ has the form $\widetilde{x}=c(\widetilde{T}) \widetilde{g}$, where

$$
\int_{-\infty}^{\infty} \frac{|c(\lambda)|^{2}}{1+\lambda^{2 n}} d \lambda
$$

converges for some $n$.

The relations

$$
\widetilde{x}=\left[\left(\int_{-n}^{n} c(\lambda) d\left(\widetilde{E}_{\lambda}-\widetilde{E}_{0}\right) \widetilde{g}\right)\right]=\left[\left(\frac{1}{\sqrt{2 \pi}} \int_{-n}^{n} c(\lambda) e^{-i \lambda t} d t\right)\right]
$$

and

$$
c(\lambda)=\frac{d}{d \lambda} \int_{0}^{\lambda} c(\mu) d \mu=\frac{d}{d \lambda}\left(\widetilde{x},\left(\widetilde{E}_{\lambda}-\widetilde{E}_{0}\right) \widetilde{g}\right)=\frac{1}{\sqrt{2 \pi}} \frac{d}{d \lambda}\left(\widetilde{x}, \frac{e^{-i \lambda t}-1}{-i t}\right)
$$

which when $\widetilde{x}$ is in $L^{2}$ correspond to the Fourier-Plancherel transformation and its inverse, provide an extension of this transformation and its inverse to the generalized elements with respect to $\Lambda$ and $P$ in $L^{2}(-\infty, \infty)$. The classifications $c(\widetilde{T}) \widetilde{g} \in G_{P} \Leftrightarrow c \in G_{1}$ and $c(\widetilde{T}) \widetilde{g} \in L^{2} \Leftrightarrow c \in L^{2}$ correspond to well-known theorems about Fourier transforms.

ExAmple 4. Let $T_{1}$ and $T_{2}$ be the differential operator $i(d / d t)$ restricted to those absolutely continuous functions $x$ in $L^{2}(-a, a)$ which 
have their derivative in this space, and satisfy $x(a)=-x(-a)$ and $x(a)=x(-a)$ respectively. Both transformations are self-adjoint and have a discrete and simple spectrum. The characteristic values for $T_{1}$ are $\lambda_{n}=(\pi / a)(n+(1 / 2))$ and for $T_{2}$ they are $\mu_{n}=(\pi / a) n, n=$ $0, \pm 1, \pm 2, \cdots$. The corresponding normalized characteristic vectors are $\varphi_{n}=(1 / \sqrt{2 a}) e^{-i \lambda_{n} t}$ and $\psi_{n}=(1 / \sqrt{2 a}) e^{-i \mu_{n} t}$. Both $G_{T_{1}}$ and $G_{T_{2}}$ are nuclear. The second of these spaces is the space $\hat{K}(a)$ of periodic infinitely differentiable functions of period $2 a$ [5, Ch. I, §3.6].

To illustrate Theorem 3 we note that any $\tilde{x}=\sum c_{\nu} \varphi_{\nu}$ in $\widetilde{G}_{T_{1}}$ can be written as $\widetilde{x}=T_{1}^{n} x$, where $x=\sum c_{\nu} \lambda_{\nu}^{-n} \varphi_{\nu}$ is in $H$ for sufficiently large $n$, and every $\tilde{x}=\sum c_{\nu} \psi_{\nu}$ in $\widetilde{G}_{T_{2}}$ has the form $\tilde{x}=T_{2}^{n} x_{1}+x_{2}$ where $x_{2}=c_{0} \psi_{0}$ is in $G_{T_{2}}$ and $x_{1}=\sum_{\nu \neq 0} c_{\nu} \lambda_{\nu}^{-n} \psi_{\nu}$ is in $H$.

Finally, we make the observation that as a rule $\widetilde{T}_{1}$ and $\widetilde{T}_{2}$ give different results even if they operate on a differentiable function $x$ in $L^{2}$ and that neither $\widetilde{T}_{1} x$ nor $\widetilde{T}_{2} x$ is equal to $i(d x / d t)$. We have, in fact,

$$
\widetilde{T}_{1} x=-i(x(a)+x(-a)) \tilde{\delta}_{a}+i \frac{d x}{d t}
$$

and

$$
\widetilde{T}_{2} x=-i(x(a)-x(-a)) \tilde{\delta}_{a}+i \frac{d x}{d t} .
$$

In general, the extension to $L^{2}$ of a differential operator with boundary conditions remembers these conditions. This fact is useful in some applications, and it supports the statement that $\widetilde{T}$ is a natural extension of $T$.

The author is indebted to the referee for improvements in the presentation and terminology of the note concerning completeness on page 51 .

\section{REFERENCES}

1. N. I. Achieser, and I. M. Glasmann, Theorie der linearen Operatoren im HilbertRaum, Akademie-Verlag, Berlin, 1960.

2. A. Friedman, Generalized Functions and Partial Differential Equations, PrenticeHall, 1963.

3. I. M. Gelfand, and G. E. Schilow, Verallgemeinerte Funktionen II, VEB, Berlin, 1962.

4. —, Verallgemeinerte Funktionen III, VEB, Berlin, 1964.

5. I. M. Gelfand, and N. J. Wilenkin, Verallgemeinerte Funktionen IV, VEB, Berlin, 1964.

6. M. Giertz, On the solutions in $L^{2}(-\infty, \infty)$ of $y^{\prime \prime}+(\lambda-q(x)) y=0$ when $q$ is rapidly increasing, Proc. London Math. Soc. (3) 14 (1964), 53-73.

7. — On the expansion of certain generalized functions in series of orthogonal functions, Proc. London Math. Soc. (3) 14 (1964), 45-52. 
8. J. Korevaar, Pansions and the theory of Fourier transforms, Trans. Amer. Math. Soc. 91 (1959), 53-101.

9. M. J. Lighthill, An Introduction to Fourier Analysis and Generalised Functions, Cambridge, 1958.

10. J. Mikusiński, Sur la méthode de généralisation de M. Laurent Schwartz et sur la convergence faible, Fund. Math. 35 (1948), 235-239.

11. J. Mikusiński, and R. Sikorski, The elementary theory of distributions (I), Rozprawy Matematyczne XII, 1957.

12. - The elementary theory of distributions (II), Rozprawy Matematyczne XXV, 1961.

13. J. B. Miller, Normed spaces of generalized functions, Compositio Mathematica

(2) 15 (1963), 127-146.

14. A. Pietsch, Über die Erzeugung von $(F)$-Räumen durch selbstadjungierte Operatoren, Math. Annalen 164 (1966), 219-224.

15. F. Riesz and B. Sz.-Nagy, Functional Analysis, Ungar, 1955.

16. L. Schwartz, Theorie des distributions, Herman, 1957.

17. V. Šedivá-Trnková, Non-F-Spaces, and appeared in the book: General Topology and Its Relations to Modern Analysis and Algebra, Academic Press, 1962.

18. S. L. Sobolew, Méthode nouvelle à resoudre le problème de Cauchy pour les équations linéares hyperboliques normales, Mat. sbornik 1 (1936), 39-72.

19. A. E. Taylor, Introduction to Functional Analysis, Wiley, 1958.

20. G. Temple, Generalized functions, Proc. Roy. Soc. (A) 228 (1955), 175-190.

21. O. Widlund, On the expansion of generalized functions in series of Hermite functions, Kungl. Tekn. Högsk. Handl., Stockholm, No. 173, 1961.

22. Zhang Gong-Zhing, Theory of distributions of $S$ type and pansions, Chin. Math. 4 (1963), 211-221.

Received June 20, 1966. This research was partially supported by the Swedish Council for Applied Research, and the Swedish Natural Science Research Council, grants No. 4678 and 5685 .

ROYAL INSTITUTE OF TECNOLOGY, STOCKHOLM

AND

UNIVERSity OF CALIFORNIA, LOS ANGELES 



\section{PACIFIC JOURNAL OF MATHEMATICS}

\section{EDITORS}

\section{H. ROYDEN}

Stanford University

Stanford, California

J. P. JANS

University of Washington

Seattle, Washington 98105

\section{J. DUGUNDJI}

Department of Mathematics

Rice University

Houston, Texas 77001

RICHARD ARENS

University of California

Los Angeles, California 90024

ASSOCIATE EDITORS

E. F. BECKENBACH

B. H. NeumanN

F. WoLF

K. YosidA

\section{SUPPORTING INSTITUTIONS}

\author{
UNIVERSITY OF BRITISH COLUMBIA \\ CALIFORNIA INSTITUTE OF TECHNOLOGY \\ UNIVERSITY OF CALIFORNIA \\ MONTANA STATE UNIVERSITY \\ UNIVERSITY OF NEVADA \\ NEW MEXICO STATE UNIVERSITY \\ OREGON STATE UNIVERSITY \\ UNIVERSITY OF OREGON \\ OSAKA UNIVERSITY \\ UNIVERSITY OF SOUTHERN CALIFORNIA
}

\author{
STANFORD UNIVERSITY \\ UNIVERSITY OF TOKYO \\ UNIVERSITY OF UTAH \\ WASHINGTON STATE UNIVERSITY \\ UNIVERSITY OF WASHINGTON \\ AMERICAN MATHEMATICAL SOCIETY \\ CHEVRON RESEARCH CORPORATION \\ TRW SYSTEMS \\ NAVAL ORDNANCE TEST STATION
}




\section{Pacific Journal of Mathematics}

\section{Vol. 23, No. 1 \\ March, 1967}

M. J. C. Baker, A spherical Helly-type theorem ................... 1

Robert Morgan Brooks, On locally m-convex*-algebras.............. 5

Lindsay Nathan Childs and Frank Rimi DeMeyer, On automorphisms of separable algebras ...................................

Charles L. Fefferman, A Radon-Nikodym theorem for finitely additive set

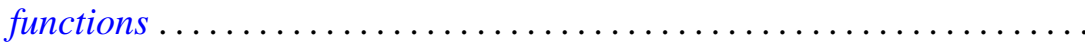

Magnus Giertz, On generalized elements with respect to linear

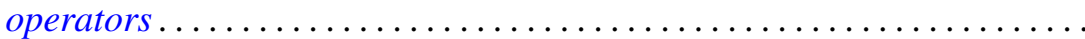

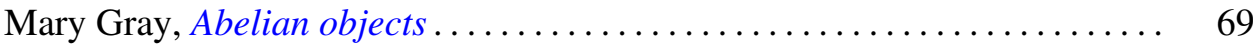

Mary Gray, Radical subcategories.............................. 79

John A. Hildebrant, On uniquely divisible semigroups on the two-cell . . . . . 91

Barry E. Johnson, AW*-algebras are $\mathrm{QW}^{*}$-algebras ............... 97

Carl W. Kohls, Decomposition spectra of rings of continuous functions . . . . 101

Calvin T. Long, Addition theorems for sets of integers .............. 107

Ralph David McWilliams, On $w^{*}$-sequential convergence and quasi-reflexivity ................................... 113

Alfred Richard Mitchell and Roger W. Mitchell, Disjoint basic

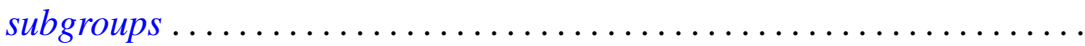

John Emanuel de Pillis, Linear transformations which preserve hermitian and positive semidefinite operators .

Qazi Ibadur Rahman and Q. G. Mohammad, Remarks on Schwarz's lemma

Neal Jules Rothman, An $L^{1}$ algebra for certain locally compact topological semigroups ...

F. Dennis Sentilles, Kernel representations of operators and their adjoints ...

D. R. Smart, Fixed points in a class of sets

K. Srinivasacharyulu, Topology of some Kähler manifolds

Francis C.Y. Tang, On uniqueness of generalized direct decompositions .

171 Albert Chapman Vosburg, On the relationship between Hausdorff dimension and metric dimension . 